\title{
Differentiated Services Based Priority Dropping and Its Application to Layered Video Streams
}

\author{
Markus Fidler \\ Chair of Computer Science IV, RWTH Aachen, \\ Ahornstr. 55, 52074 Aachen, Germany \\ fidler@i4.informatik.rwth-aachen.de
}

\begin{abstract}
In this paper we report on an implementation and evaluation of Internet priority dropping schemes and their application to layered video transmissions. The incremental decoding of each of the different layers of such an hierarchical video stream leads to an enhancement of the video quality, whereas the absence of a layer renders the receipt of higher layers useless. Thereby the layers have a specific order of precedence, which reflects their importance on the video quality and on the decoding process. We accommodate this hierarchy by mapping the video layers on different traffic classes implemented in a Differentiated Services network. We present a thorough evaluation of these schemes and we demonstrate the performance gain, if different Quality of Service classes for the transmission of the different layers are applied. Further on we address the interaction between non-responsive video flows and responsive streams and show how fairness can be supported by this approach.
\end{abstract}

\section{Introduction}

The convergence of telecommunication systems and data networks requires services beyond what is currently provided as Best-Effort (BE) service by the Internet. Especially real-time audio and video streams have significantly different traffic properties and requirements than common web or file transfer traffic. For example for high quality video-conferencing Quality of Service (QoS) parameters like throughput, delay, and drop rate are critical for the performance. On the other hand these applications commonly use the User Datagram Protocol (UDP) and the Real-time Transmission Protocol (RTP) 24. They are often not responsive to congestion and tend to steal capacity from responsive Transmission Control Protocol (TCP) flows. Thus also application adaptivity and TCP-friendly rate control are required.

Since the first Internet congestion collapse a number of mechanisms that address congestion have been developed. These are mainly TCP slow start and congestion avoidance [1], which are supported in the network by Random Early Detection (RED) [7]. But also for non TCP flows, effort is made to standardize congestion control 8], both to enhance network performance and to increase fairness, if these flows compete with responsive TCP flows. 
Congestion is in the absence of Explicit Congestion Notification (ECN) [20] indicated by packet drops. If packets are lost, mainly three options exist for a real-time audio or video application: Dropped packets can simply be ignored, thus leading to a quality reduction, Forward Error Correction (FEC) 22] can be applied to recover from further lost packets, or Automatic Repeat Request (ARQ) techniques can be used for retransmissions 15 18. FEC and ARQ mechanisms do increase the senders data rate. In order to compensate for this effect and to reduce congestion, a rate adaptation must be performed 27. An option is to apply FEC or ARQ selectively to protect only the more important parts of a stream 1825. An hierarchical separation of video data into a set of layers 4,13] allows for the desired differentiation in terms of importance. Layered video also serves in this context for coarse congestion control schemes [21], and multi-cast scenarios with heterogeneous clients [16].

Apart from a protection by FEC or ARQ, the network can protect streams or sub-streams by applying priority dropping. A comparison of uniform and priority dropping schemes for layered video is given in [2]. Analytic and simulative results are shown for performance and incentive properties. The authors find that in case of the applied utility functions priority dropping does result in a performance gain, but they conclude that in the absence of proven utility models no definitive answer can be given. Nevertheless we have shown in simulations that in terms of the Signal-to-Noise Ratio (SNR) priority dropping performs better than uniform dropping [6]. Further on, we assume that instead of protecting parts of a stream by FEC or ARQ, it is better to apply different drop precedence level implemented by the network. Doing so also ensures the transmission of the more important parts of the stream, while less important parts can be dropped due to congestion. Moreover the disadvantages of FEC and ARQ like an increased data rate and an additional play out delay are not immanent to this approach.

In the future Internet an implementation of different drop rates can be based on the precedence field in the Internet Protocol (IP) Type of Service (ToS) field. A more advanced implementation of different drop precedence classes can apply Differentiated Services (DS) [3], which is the most recent approach of the Internet Engineering Task Force (IETF) towards QoS. The scalability issues encountered with the formerly proposed Integrated Services architecture are addressed by DS by a Class of Service (CoS) aggregation of individual flows to classes. The sender can mark packets to be of a certain priority in the RTP 19 header or, if the sender is aware of the QoS mechanisms provided by the network, it can perform the relevant marking, for example set the IP ToS field. It has to be noted that also in Asynchronous Transfer Mode (ATM) networks two drop priorities, which can be assigned to critical and non-critical data of a video stream, based on the ATM Cell Loss Priority (CLP) bit, exist [17.

In the remainder of this paper we focus on a real-time video application like video-conferencing with data rates in the range of a few tens of $\mathrm{kb} / \mathrm{s}$ upto $5 \mathrm{Mb} / \mathrm{s}$, and delay requirements of about $100 \mathrm{~ms}$ [14. It is organized as follows: We give an overview on video coding in Sect. 2 and on DS in Sect. 3. In Sect. 4 we show our experimental setup and the obtained results and conclude in Sect. 5. 


\section{$2 \quad$ Video Coding}

Common video coding standards like H.263+ 4] and MPEG-4 [13] implement video data compression by applying quite similar means. The data reduction is achieved by reducing redundancy and by dropping information, which is of less importance for the human visual system. This is performed concerning spatial information on single pictures (intra-frame), and concerning temporal information between different consecutive pictures (inter-frame). Temporal redundancy between consecutive frames is addressed by predicting pictures from earlier ones. Two types of predicted frames are used, forward predicted P-frames and bidirectionally predicted B-frames. In addition to the predicted frames intra-coded I-frames exist, which are encoded independently. Figure 1 shows a simplified video encoder consisting of Discrete Cosinus Transform (DCT), Quantizer (Q), and Entropy Encoder (EE) used for I-frames and P-frame difference pictures, and a similar decoder with Motion Estimation (ME) for the generation of the $\mathrm{P}$-frames motion vectors.

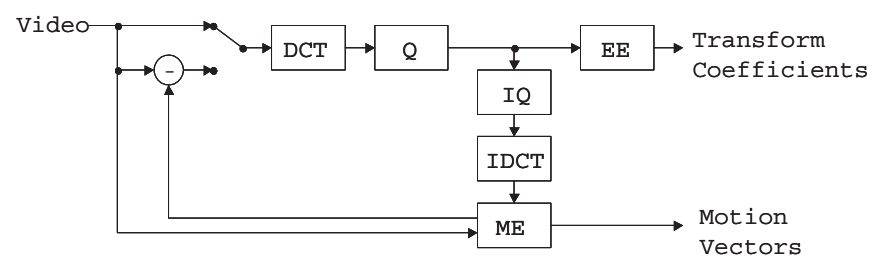

Fig. 1. Video Encoder

The quality of an encoded stream, the compression gain, and thus the data rate depend on the applied quantization parameter. The Peak $(\mathrm{P})$ SNR according to 1 that is realized with the tmn H.263+ enocder is shown for different data rates in the top curve in Fig. 3 for the "apple" sequence. The encoder input is Quarter Common Intermediate Format (QCIF) with $i=176 \times j=144$ pixels with 8 bit depths and a luminance $(\mathrm{Y})$ chrominance $(\mathrm{U}, \mathrm{V})$ sub-sampling of 4:1:1.

$$
P S N R=\frac{i \cdot j \cdot Y_{\max }^{2}}{\sum_{i} \sum_{j}\left(Y_{\text {orig }}(i, j)-Y_{\text {dec }}(i, j)\right)^{2}}
$$

Current video coders allow for a hierarchical coding in a set of layers, which are classified into a base layer and a number of enhancement layers. The base layer contains all information that is necessary to display the video with a comparably low quality. On top of the base layer an incremental decoding of the enhancement layers is possible, whereas each successive layer improves the quality of the decoded stream. Three options of hierarchical encoding are defined in the H.263+ standard [4]. These are temporal, SNR, and spatial scalability as 
shown in Fig. 22, The arrows indicate the direction of prediction. In case of layered video it can be distinguished between forward prediction within one layer and upward prediction between the layers. In addition the MPEG standards allow for the option of data partitioning.
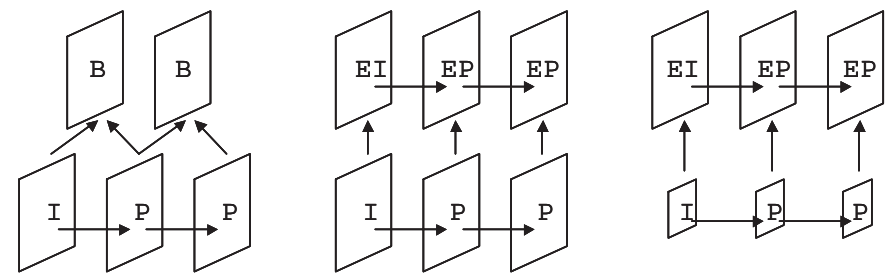

Fig. 2. Temporal, SNR, and Spatial Scalability

- Temporal scalability is achieved deploying the disposable nature of B-frames. Due to the high compression gain of B-frames the data rate of the enhancement layer is comparably low, but B-frames increase the play out delay.

- Signal-to-Noise Ratio scalability permits the refinement of the video in terms of the SNR. The quantization parameter of the encoder can be set independently for each layer and thus enables an encoding of the layers with independent target data rates, and an increasing SNR.

- Spatial scalability is achieved by encoding the frames with a low resolution for the base layer and with a higher resolution for the enhancement layers. Spatial scalability is apart from an up-sampling procedure implemented by the same means as SNR scalability.

An example of the PSNR of an encoded sequence applying SNR scalability is given in Fig. 4. In Fig. 5 and 6 a base and the corresponding enhancement layer picture are shown. Both sub-streams of this example are encoded with a rate of 30 frames per second and a data rate of about $50 \mathrm{~kb} / \mathrm{s}$.

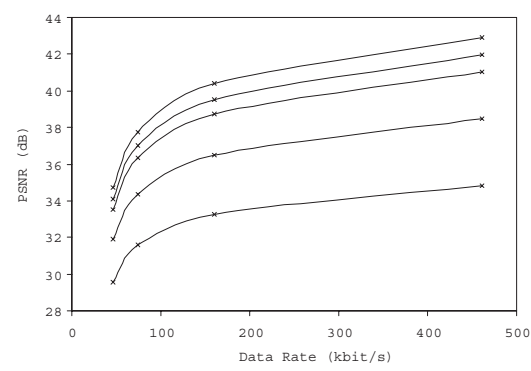

Fig. 3. Drop Rates $\{0 ; 0.01 ; 0.02 ; 0.05 ; 0.1\}$

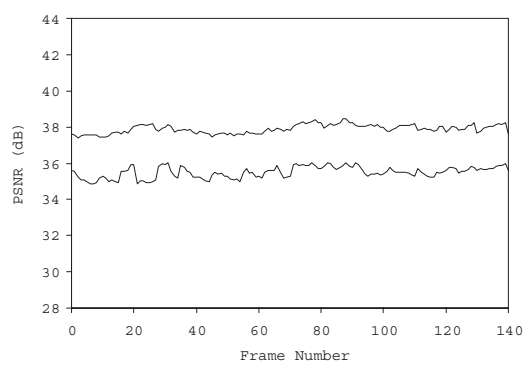

Fig. 4. Base/Enhancement Layer 


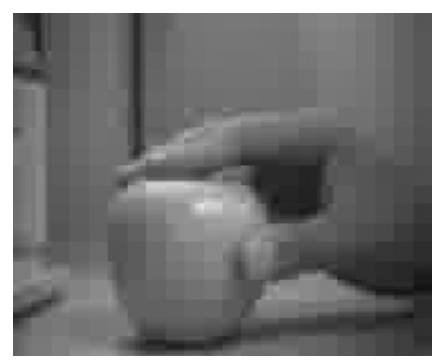

Fig. 5. Base Layer

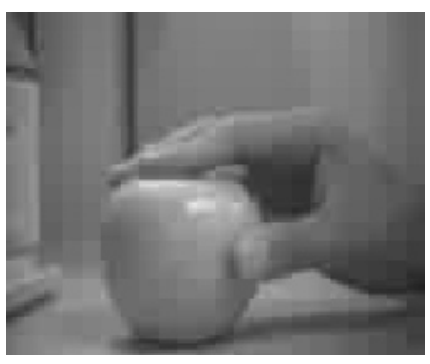

Fig. 6. Base+Enhancement Layer

Though video applications are considered to be loss tolerant [14, packet drops interfere with the decoding process and have adverse effects on the PSNR as shown in Fig. 3 Especially the effects of error propagation have to be considered. Due to the predictive encoding, errors propagate through the frame sequence up to the next synchronization point, namely the next I-frame. Thereby the robustness of the stream is directly influenced by the frame distance between consecutive I-frames $n$, whereas $n=10$ in Fig. 3. To address these effects we describe frame loss and error propagation with a simple analytical model based on only two layers. A more general approach based on utility functions is given in [2], with the drawback that the generality limits the significance of the results. Equation 2 gives the probability of an error free decoding of a base layer frame $i$ for a base layer frame loss probability $p_{b}$. The corresponding probability for enhancement layer frames is given in 3 , with the respective loss probability $p_{e}$.

$$
\begin{aligned}
& q_{b}(i)=\left(1-p_{b}\right)^{((i \bmod n)+1)} \\
& q_{e}(i)=\left(\left(1-p_{b}\right) \cdot\left(1-p_{e}\right)\right)^{((i \bmod n)+1)}
\end{aligned}
$$

With $p_{b}=p_{e}=p$, 2 and 3 describe the special case of uniform dropping. For reasons of simplicity we further on assume that the base and enhancement layer are encoded with a similar frame and data rate, which can for example be achieved by SNR scalability. Under the constraint $p=\left(p_{b}+p_{e}\right) / 2$, which ensures that base layer frames can only be protected at the cost of a corresponding increase of the enhancement layer drop rate, a comparison of uniform and priority dropping can be investigated. We formulate the optimization problem in 4 for one Group of Pictures (GoP), with the weight $w_{b}$ denoting the quality gain of an error free decoding of a base layer frame and $w_{e}$ giving a similar weight for an enhancement layer frame. The decoding probabilities $p_{b}$ and $p_{e}$ are statistically dependent, such that the decoding of an enhancement layer frame implies the error free decoding of the relevant base layer frame. Therefore the resulting weight $w=\left(w_{e}-w_{b}\right)$ is applied for the enhancement layer. It can be assumed that $w_{b}>\left(w_{e}-w_{b}\right)$ in terms of PSNR, as can be seen from Fig. 3.

$$
\max \left(\frac{w_{b}}{n} \sum_{i=0}^{n-1} q_{b}(i)+\frac{w_{e}-w_{b}}{n} \sum_{i=0}^{n-1} q_{e}(i)\right)
$$


Since $p_{b}+p_{e}=2 p \ll 1$ we apply the approximation $\left(1-p_{b}\right)\left(1-p_{e}\right) \approx 1-2 p$. Thereby the term that denotes the quality gain of a successful decoding of the enhancement layer does not depend on the individual drop probabilities of base and enhancement layer, whereas the term that denotes the quality gain of a successful decoding of the base layer depends only on $p_{b}$. The maximum according to 4 is then found for $p_{b}=0$ and $p_{e}=2 p$, thus indicating the advantage of priority compared to uniform dropping.

\section{Differentiated Services}

A DS network [3] is composed of ingress respective egress nodes at the edges and core nodes within the DS domain. At ingress nodes a classification of the traffic into the different classes, which are supported within the domain, is performed. The respective class or behavior aggregate is marked as DS Code Point (DSCP), which supercedes the IP ToS field. Apart from this marking, the incoming traffic can be metered against some defined traffic characteristics usually by applying a token bucket, it can be policed or dropped, if certain traffic parameters are exceeded, and traffic shaping can be applied, to limit traffic bursts of certain classes that enter the DS domain, for example by applying a leaky bucket.

Within the DS domain each DSCP is mapped on a Per Hop Behavior (PHB). A share of the link capacities is reserved for each of these PHB. The PHB specify the service that is applied for the pertaining packets on their way to the next hop. So far one PHB and one PHB group have been defined. The single PHB is the so called premium service Expedited Forwarding (EF) [9], which provides low loss, low delay, and low delay jitter. A recommended implementation of EF is Priority Queuing (PQ). The PHB group is Assured Forwarding (AF) 9] and consists of four independent forwarding classes, whereas each class allows for a differentiation of upto three levels of drop precedence. Usually the AF classes are realized by a Weighted Fair Queuing (WFQ) environment, whereas Multiple (M)-RED implements the different levels of drop precedence.

AF packets can be marked by an ingress marker as green, yellow, or red depending on whether they conform to some specified traffic characteristics or not. A marking can be performed by a Single Rate Three Color Marker (SRTCM) [10. The SRTCM is based on a token bucket with a Committed Information Rate (CIR), a Committed Burst Size (CBS) and an Excess Burst Size (EBS). A packet is marked green, if it does not exceed the CBS, yellow if it exceeds the CBS but not the EBS, and red otherwise. An alternative implementation is the use of a Two Rate Three Color Marker (TRTCM) [1], which marks a packet red, if it exceeds a Peak Information Rate (PIR), yellow, if it does not exceed the PIR but the CIR and green otherwise. Both of the TCM can be used either in the color-blind mode, in which an incoming stream is assumed to be uncolored, or in the color-aware mode, if packets are pre-colored for example by an upstream DS domain or by the sending host itself. Within the DS domain the color of the packet determines the mapping of the packet on the applied drop precedence. 
Besides the EF and AF PHB a so-called Scavenger Service (SS) [26] was proposed in the DS environment recently. This service forms a less than BE service, which allows applications to utilize unused capacity, while the BE traffic is protected by assigning only a small weight of the link capacity to the SS. The SS thereby can allow for the transmission of high data rate and even unresponsive flows ideally without interfering with the BE service. A typical SS implementation is WFQ with a minimum link share that is configured to $1 \%$.

Figure 7 and 8 show the DS configuration of a core router. The EF, one of the four possible $\mathrm{AF}$, the BE, and the $\mathrm{SS}$ queues are shown in the model. Besides two possible M-RED configurations are given, one with only two drop precedence level for a differentiation of green and red packets within an AF class, and one that in the absence of DS can be applied to the BE class, if a IP ToS precedence marking is supported. In addition to the queues that apply to each of the different services, Fig. 7 also shows the Layer 2 (L2) queue that is located on the outgoing interface card of the router. This queue is required to allow for a smooth operation of the interface and has to be configured carefully, since it can add further delay and delay jitter to all DS classes [5].

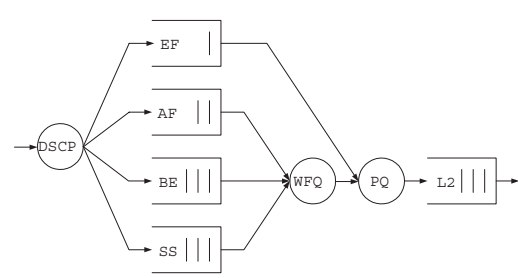

Fig. 7. DS Queuing and Scheduling
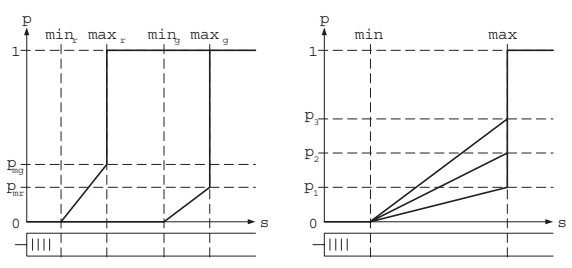

Fig. 8. MRED Configurations for AF and $\mathrm{BE}$

\section{Experimental Studies}

We report on experiments made with a video application that utilizes different QoS classes for the transmission of layered video streams within a DS testbed. The option of SNR scalability of the H.263+ tmn encoder version 3.2 is used to generate only two layers, in order to minimize the overhead and because we think that a DS deployment with a limited number of traffic classes is more feasible. Our video transmission tool is capable of performing a marking of the packets, by setting the IP ToS or the DSCP field. Alternatively a marking according to [19] can be made in the RTP header, or by dividing the stream into sub-streams with different port fields, whereas both require a corresponding setting of the DSCP at the DS domains ingress node. In addition to the video transmission tool, we make use of the common UDP BE traffic generator gen-send/gen-recv to create congestion, and the TCP traffic generator ttcp to evaluate effects on TCP congestion control. Our testbed shown in Fig. 9 consists of four CISCO 
7200 routers connected either by OC3 ATM, or Gigabit Ethernet links, whereas end systems are connected by switched Fast Ethernet. One of the ATM links is configured as a bottleneck link to $60 \mathrm{Mb} / \mathrm{s}$, which corresponds to a netto rate of about $48 \mathrm{Mb} / \mathrm{s}$ after subtracting the ATM overhead.

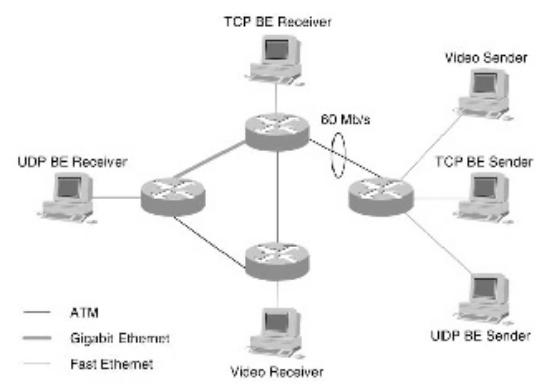

Fig. 9. DS Testbed in Jülich, donated by Cisco Systems.

The router configuration used in the tests implements an EF class by PQ, an AF class with a WFQ share of $5 \%$, the SS with $1 \%$, and the BE class with the remaining capacity. The AF class supports green and red marked packets with an M-RED implementation applying the thresholds $\min _{r}=16, \max _{r}=3^{2}$, min $_{g}=48$, and $\max _{g}=64$, and the maximum probabilities $p_{m r}=p_{m g}=0.1$. In case of pure BE experiments, a setting of the IP ToS precedence field is mapped onto an M-RED implementation with $\min =64, \max =128, p_{1}=0, p_{2}=0.5$, and $p_{3}=1$, which supports a high, medium, and low drop precedence. The L2 queue is set to 4 Maximum Transmission Units (MTU), here $4 \cdot 1500 \mathrm{~B}$.

We made experiments with a mapping of video layers to traffic classes according to Tab. 1 Simple BE experiments have been made as a comparison. No differentiation between $\mathrm{EF}$ and $\mathrm{AF}$ is made in scenario 5 and 6 , since both classes can be used to build the guaranteed rate service that is applied here, whereas significant differences exist in terms of delay and delay jitter [23].

Table 1. Mapping of Video Layers to Traffic Classes

\begin{tabular}{|c|c|c|c|c|}
\hline Sc. & Type & Dropping & Base Layer & Enhancement Layer \\
\hline 1 & BE & Uniform & \multicolumn{2}{|c|}{ Medium Precedence } \\
\hline 2 & DS & Uniform & \multicolumn{2}{|c|}{ AF } \\
\hline 3 & BE & Priority & High Precedence & Low Precedence \\
\hline 4 & DS & Priority & AF Green & AF Red \\
\hline 5 & DS & Priority & EF $/ A F$ & BE \\
\hline 6 & DS & Priority & EF $/ A F$ & SS \\
\hline
\end{tabular}


The PSNR of a repeated transmission of the "apple" sequence obtained from measurements in the testbed is shown for the different scenarios of Tab. 1 in Fig. 10, 15, whereas BE congestion is created by a bursty and non-responsive $47 \mathrm{Mb} / \mathrm{s}$ UDP flow that starts after the transmission of 140 frames for the duration of 280 frames. Figure 10 shows the transmission of only the base layer in the BE class. During congestion base layer frames are dropped and the PSNR of the received stream oscillates noticeable. Thus, the quality at the receiver is degraded both, by the lower PSNR and also by the fluctuations of the PSNR, which are disturbing for the human visual system. The same applies if the sender transmits the base and the enhancement layer in the BE class as shown in Fig. 11, apart from the higher PSNR during periods without congestion. Similar experiments have been carried out with the AF implementation. The main difference is an AF reservation for the base layer. This reservation, as long as it is not exceeded, ensures the transmission of the base layer even during BE congestion as shown in Fig. 12 If the sender transmits base and enhancement layer, the AF class is oversubscribed and AF packet loss occurs during congestion. This results in similar effects as in the BE class as can be seen in Fig. 13

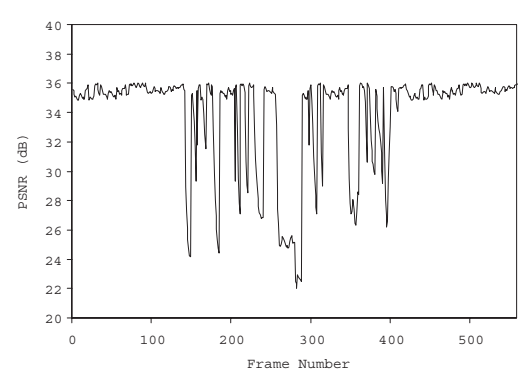

Fig. 10. Base Lay. - Medium Prec.

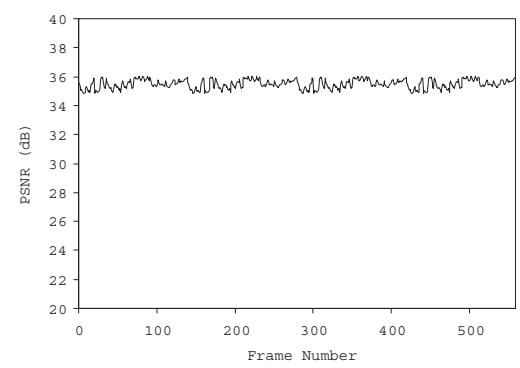

Fig. 12. Base Lay. - AF

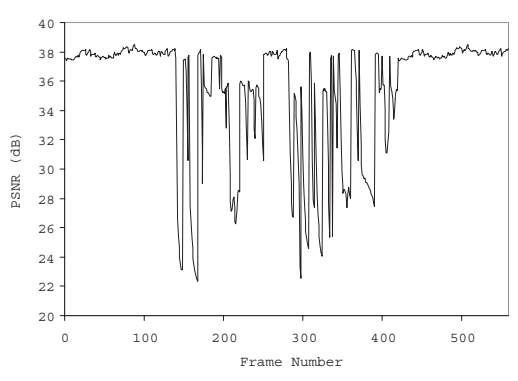

Fig. 11. Base/Enh. Lay. - Medium Prec.

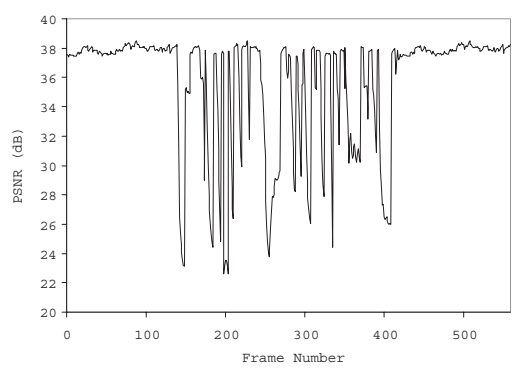

Fig. 13. Base/Enh. Lay. - AF

Figure 14 shows results from the BE video transmission, but now with a mapping of the base layer to a high and the enhancement layer to a low drop precedence. Congestion is created by a stream with medium drop precedence. In this scenario congestion only affects the enhancement layer. Base layer frames are 
not dropped and thus ensure a certain PSNR. Further on the PSNR oscillations are limited to a range between the PSNR of the base and the PSNR of the enhancement layer. Figure 15 shows similar results from a transmission of base and enhancement layer in the AF class with an explicit marking of the drop precedence. Again the base layer transmission can be secured and congestion only affects the enhancment layer. The PSNR measurements obtained from scenario 5 and 6 are omitted here, because they merely show similar results as given in Fig. 15] In all of the DS experiments a sufficient amount of EF or AF capacity is reserved for a loss-free base layer transmission, whereas the transmission of the enhancement layer is affected by BE congestion.
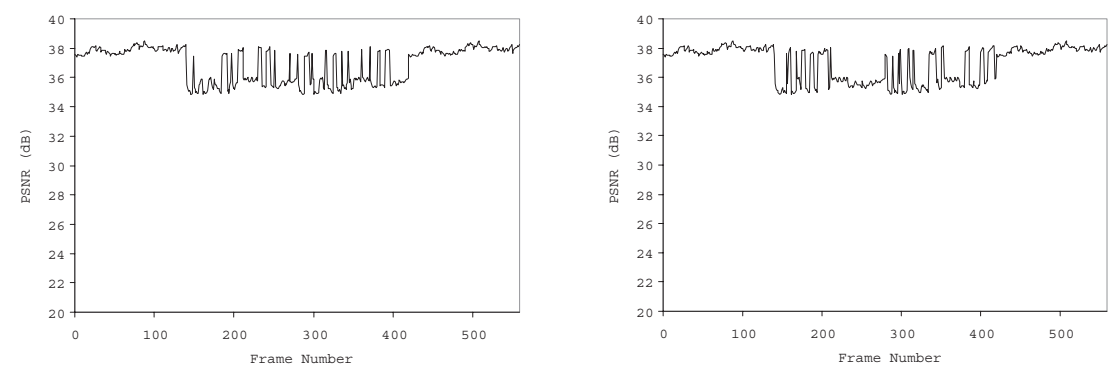

Fig. 14. Base/Enh. Lay. - High/Low Fig. 15. Base/Enh. Lay. - AF Green/Red Prec.

The effects of the video transmission on BE TCP throughput are shown in Fig. 16 19 for the respective scenarios. For these experiments a traffic mix consisting of a non-responsive $40 \mathrm{Mb} / \mathrm{s}$ UDP flow, a TCP stream limited by the application to $12 \mathrm{Mb} / \mathrm{s}$, and either only the base layer or base and enhancement layer of the video streams each of about $2.4 \mathrm{Mb} / \mathrm{s}$ is applied. The TCP stream is rate-limited by the Round-Trip-Time (RTT) and a maximum TCP window size configured by setting the socket buffer to $16 \mathrm{kB}$, whereas during periods of $15 \mathrm{~s}$ of the video base layer transmission at $10 \mathrm{~s}$ and the transmission of base and enhancement layer at $40 \mathrm{~s}$ packet loss and congestion avoidance limit TCP throughput. The base layer transmission reduces the TCP throughput by about $2.4 \mathrm{Mb} / \mathrm{s}$, independent from the scenario and the existence of a base layer reservation, only due to TCP congestion control. The same is true for an additional enhancement layer transmission in the BE class as shown in Fig. 16, and 18. In contrast, if the AF class is oversubscribed and used for the base and enhancement layer transmission or in case of an enhancement layer transmission applying the SS, the enhancement layer stream cannot preempt the TCP stream further, as shown in Fig. 17, and [19. Thus these implementation options allow for some fairness and co-existence with responsive streams.

It has to be noted that a SS applied for delay sensitive but loss tolerant RTP/UDP traffic has to fulfill different requirements than a SS for TCP traffic, which in the first place has to avoid packet loss by offering sufficient queuing 


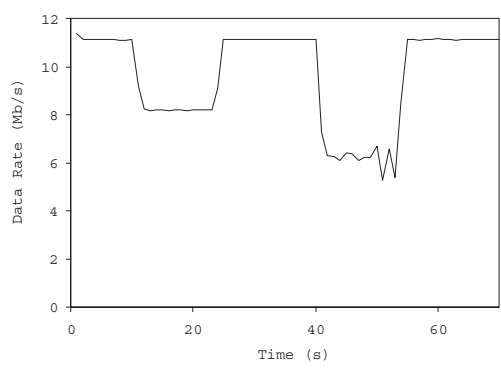

Fig. 16. BE Video vs. BE TCP

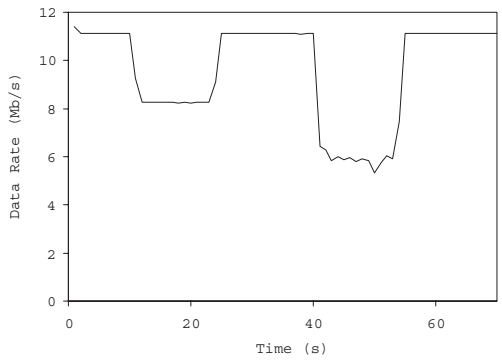

Fig. 18. EF/BE Video vs. BE TCP

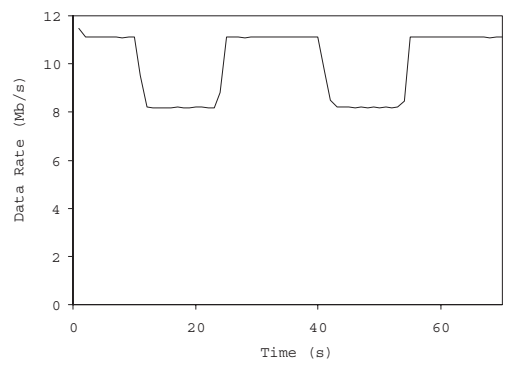

Fig. 17. AF Video vs. BE TCP

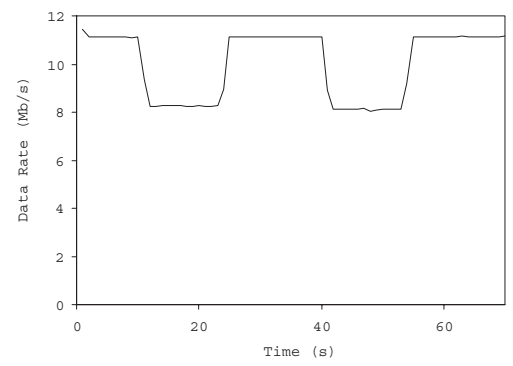

Fig. 19. EF/SS Video vs. BE TCP

space, adapted to the TCP window size. For RTP/UDP traffic rather small queues and ideally front drop have to be used, to prevent from queuing delay during periods of congestion, especially since the SS WFQ share is configured to $1 \%$. The situation is different for the AF M-RED implementation. The queuing delay that can be added by enhancement layer data, if mapped to the highest drop precedence level, can be configured for a single node by the setting of $\max _{r}$, for a certain AF WFQ share. Further on AF traffic can be limited at DS ingress nodes and the impacts on BE TCP streams can be controlled by the assigned WFQ share, wherefore we recommend the AF based implementation.

\section{Conclusions}

In this paper we have shown how hierarchical video transmissions can be supported by priority dropping schemes. We have addressed the options of the H.263+ standard to generate such streams and we have shown several possible implementations of different drop probabilities in the future Internet, by applying either a BE precedence marking or the different PHB of DS. We have performed a thorough evaluation of our implementation of the proposed framework. Significant performance benefits and also the impacts on responsive flows have been shown. The different AF drop precedence level have been identified as a prime implementation option. AF can be configured to avoid base layer packet loss and it allows for applying unused capacity for an enhancement layer transmission without preempting BE TCP streams in case of congestion. 


\section{References}

1. Allman, M., Paxson, V., Stevens, W.: TCP Congestion Control. RFC 2581 (1999)

2. Bajaj, S., Breslau, L., Shenker, S.: Uniform versus Priority Dropping for Layered Video. Proceedings of ACM SIGCOMM (1998)

3. Blake, S., Black, D., Carlson, M., Davies, M., Wang, Z., Weiss, W.: An architecture for Differentiated Services. RFC 2475 (1998)

4. Côté, G., Erol, B., Gallant, M.: H.263+: Video Coding at Low Bit Rates. IEEE Transactions on Circuits and Systems for Video Technology, vol. 8, no. 7 (1998)

5. Ferrari, T., Chimento, P.: A Measurement-based Analysis of Expedited Forwarding PHB Mechanisms. Proceedings of IWQoS (2000)

6. Fidler, M.: Transmission of Layered Video Streams in a Differentiated Services Network. Proceedings of AI PDCN (2002)

7. Floyd, S., Jacobson, V.: Random Early Detection Gateways for Congestion Avoidance. IEEE/ACM Transactions on Networking, vol. 1, no. 4 (1993), pp. 397-413

8. Handley, M., Padhye, J., Floyd, S.: TCP Friendly Rate Control (TFRC): Protocol Specification. Internet Draft draft-ietf-tsvwg-tfrc-03.txt (2001)

9. Heinanen, J., Finland, D., Baker, F., Weiss, W., Wroclawski, J.: An Assured Forwarding PHB. RFC 2597 (1999)

10. Heinanen, J., Guerin, R.: A single rate three color marker. RFC 2697 (1999)

11. Heinanen, J., Guerin, R.: A two rate three color marker. RFC 2698, (1999)

12. Jacobson, V., Nichols, K., Poduri, K.: An Expedited Forwarding PHB. RFC 2598, (1999)

13. Koenen, R. (Editor): Overview of the MPEG-4 Standard. ISO/IEC JTC1/SC29/ WG11 (2001)

14. Kurose, J., Ross, K.: Computer Networking - A Top-Down Approach Featuring the Internet. Addison Wesley (2001) p. 80

15. Leon, D., Varsa, V.: RTP retransmission framework. Internet Draft draft-ietf-avtrtp-selret-03 (2001)

16. McCanne, S., Jacobson, V., Vetterli, M.: Receiver-driven Layered Multicast. Proceedings of ACM SIGCOMM (1996)

17. McDysan, D.: QoS \& Traffic Management in IP \& ATM. McGraw-Hill (2000)

18. Miyazaki, A. et al.: RTP Payload Formats to Enable Multiple Selective Retransmission. Internet Draft draft-ietf-avt-rtp-selret-03 (2001)

19. Polk, J.: RTP Header Extension for Communications Resource Priority. Internet Draft draft-polk-avt-rtpext-res-pri-00 (2001)

20. Ramakrishnan, K., Floyd, S., Black, D.: The Addition of Explicit Congestion Notification (ECN) to IP. RFC 3168 (2001)

21. Rejaie, R., Handley, M., Estrin, M.: Quality Adaptation for Congestion Controlled Video Playback over the Internet. Proceedings of ACM SIGCOMM (1999)

22. Rosenberg, J., Schulzrinne, H.: An RTP Payload Format for Generic Forward Error Correction. RFC 2733 (1999)

23. Sander, V., Fidler, M.: Evaluation of a Differentiated Services based Implementation of a Premium and an Olympic Service. Submitted to IWQoS (2002)

24. Schulzrinne, H., Casner, S., Frederick, R., Jacobson, V.: RTP: A Transport Protocol for Real-Time Applications. RFC 1889 (1996)

25. Tan, W., Zakhor, A.: Video Multicast using Layered FEC and Scalable Compression. IEEE Trans. on Circuits and Systems for Video Tech., vol. 11, no. 3 (2001)

26. Teitelbaum, B.: Future Priorities for Internet2 QoS. www.internet2.edu/qos/wg/ papers/qosFuture01.pdf (2001)

27. Wang, X., Schulzrinne, H.: Comparison of Adaptive Internet Multimedia Applications. IEICE Transactions on Communications June (1999) 